\title{
Barrier Distributions as a Tool to Investigate Fusion and Fission
}

\author{
M. Dasgupta, ${ }^{a}$ D.J. Hinde ${ }^{a}$, J.R. Leigh $^{a}$ and K. Hagino ${ }^{\mathrm{b}}$ \\ aDepartment of Nuclear Physics, Research School of Physical Sciences and Engineering, \\ The Australian National University, Canberra ACT 0200, Australia \\ b Department of Physics, Tohoku University, Sendai 980-77, Japan
}

The recent availability of precisely measured fusion cross-sections has enabled the extraction of a representation of the distribution of barriers encountered during fusion. These representations, obtained from a variety of reactions, provide a direct observation of how the structure of the fusing nuclei changes the inter-nuclear potential landscape, thus affecting the fusion probability. Recent experiments showing the effects of static quadrupole and hexadecapole deformation, single- and double-phonon states, transfer of nucleons between two nuclei, and high lying excited states are reviewed. The application of these concepts to the explanation of the anomalous fission-fragment anisotropies observed following reactions with actinides is discussed.

\section{INTRODUCTION}

In the simplest fusion model, two nuclei are assumed to fuse once they have penetrated the one-dimensional real potential barrier resulting from the sum of the repulsive Coulomb and centrifugal potentials, and the attractive, short range nuclear potential. However, for reactions involving heavy ions, the measured fusion cross-sections were found to be significantly different from the expectations of such a model, particularly at energies below the barrier where enhancement of several orders of magnitude was observed [1,2]. The inadequacy of this model was conclusively demonstrated [3] by inverting the experimental data, which yielded unphysical inter-nuclear potentials. It was shown subsequently [4] that the observed enhancements were due to coupling of the translational motion to intrinsic degrees of freedom, e.g. rotation, or surface vibrations, a fact which is not taken into account in the single-barrier penetration model.

The role of static deformation effects [5] 7] in enhancing sub-barrier fusion can easily be visualised in a classical picture, where different orientations of the deformed target nuclei are encountered during the collision, giving rise to a distribution of barrier heights, some lower and some higher than the single-barrier. Due to the exponential dependence of the fusion probability on the barrier height, any distribution of barriers which gives a barrier at an energy lower than the single-barrier, $B_{0}$, leads to enhancement of the crosssections at energies below $B_{0}$. In general the effect of couplings to other degrees of freedom results in a change from a single-barrier penetration scenario to multi-dimensional barrier penetration. In the next section, the concept of the distribution of barriers is discussed. 
The experimental results, spanning strongly-coupled to weakly-coupled systems, and their interpretations, are presented in Secs. 3-6. The correlations and applications of these new results to other fields are discussed in Sec. 7 , followed by a summary in Sec. 8 .

\section{FUSION BARRIER DISTRIBUTIONS}

The above discussion indicates that viewing the collision process as two structureless spheres interacting only through the inter-nuclear potential is too simplistic, and coupling to other reaction channels has to be considered. Theoretical calculations including coupling to inelastic and transfer channels are usually carried out by solving a set of coupled-channels equations. The effects of such couplings, however can easily be visualised in the eigenchannel approximation, where it was shown [4 that the effects of such couplings are to replace the single-barrier by a distribution of barrier heights, thus providing an explanation of the observed disagreement [1.2] with the single-barrier penetration calculations. This improved understanding was pivotal for interpreting the experimental results. However since any coupling mechanism would give rise to enhancement, an educated guess was required for the type of coupling to be included in a given system.

This point is illustrated in Fig. 1, where three theoretical excitation functions for reactions involving different coupling schemes are shown. The coupling schemes involve coupling to a negative $Q$-value channel in Fig. 1(a), a positive $Q$-value channel in Fig. 1(b), and coupling associated with a deformed nucleus in Fig. 1(c). The discrete barriers associated (under the eigenchannel approximation) with the first two cases are shown in Fig. $\mathbb{1}(\mathrm{d})$ and (e) by the thick vertical lines with lengths indicative of the probability of encountering the barrier; the third coupling scheme will give a continuous distribution of barrier heights associated with different orientations of the deformed nucleus. Comparison with the dashed lines resulting from a single-barrier penetration calculation, shows that the enhancements at the lower energies are very similar in the three cases, despite the differences in the nature of couplings. There are significant differences in the excitation functions at other energies but the type of coupling involved is not immediately apparent from the excitation functions themselves.

A few years ago it was suggested [8] that a representation of the distribution of barrier heights in a reaction could be extracted directly from a fusion excitation function. In a classical sharp cut-off model,

$E \sigma=\sum_{\alpha} w_{\alpha} \pi R_{\alpha}^{2}\left(E-B_{\alpha}\right)$

where $E$ is the energy, $\sigma$ is the fusion cross-section, $R_{\alpha}$ is the fusion radius, $w_{\alpha}$ is the probability of encountering the barrier and $B_{\alpha}$ is the barrier energy associated with the channel index $\alpha$. In this case, $d^{2}(E \sigma) / d E^{2}$ returns the original discrete barrier distribution as a set of delta functions, centered at the barrier energies and normalised by the $w_{\alpha}$ [ [8]. When quantum mechanical penetration of the barriers is considered, the cross-sections vary smoothly in the vicinity of each barrier and $d^{2}(E \sigma) / d E^{2}$ becomes continuous, replacing the delta functions by a near Gaussian function [8]. It was also shown [9] that the function $d^{2}(E \sigma) / d E^{2}$ does indeed correspond closely to the "true" barrier distribution smoothed by the quantum tunnelling effects. 
Applying this technique to the excitation functions of Fig. 1 returns a function shown by the solid lines in the lower panels. It can be seen that they are very different for the three cases, and reveal directly where the barrier strength lies. For example, in Fig. 11(d) and (e) the solid curves are centered around the calculated discrete barrier positions (thick lines) and the heights of the gaussians reflect the probability of encountering the barrier. In the third case the solid curve coincides with the barrier distribution expected for a deformed nucleus. It should be noted that the interpretation of an experimental barrier distribution is not always as simple as suggested by the examples shown in Fig. 11. These are idealised cases and in reality several different types of coupling may be involved in a given reaction, complicating the situation. Nonetheless, the ability to see directly how barrier strength is distributed is useful in understanding the reaction process.

The function $d^{2}(E \sigma) / d E^{2}$ will be referred to as the barrier distribution in this contribution, even though it is recognised that it gives only a representation of the true barrier distribution. Also, since the experimental data are measured at discrete energies, the function $d^{2}(E \sigma) / d E^{2}$ is extracted using a point difference formula. For consistency, when comparing an experimental distribution with theory, the theoretical distribution is evaluated in an identical manner.

\section{TESTING THE CONCEPT - REACTIONS INVOLVING ROTATIONAL NUCLEI}

The concept of a fusion barrier distribution outlined above is particularly attractive, since a representation can be directly extracted from experimental data, thus reducing the uncertainty in the type of coupling schemes to be introduced to explain the experimental results. Fig. 2(a) shows the barrier distribution for the ${ }^{16} \mathrm{O}+{ }^{154} \mathrm{Sm}$ reaction, extracted from data [7] which has an experimental uncertainty of $10 \%$; a level typical for the data prior to the new generation of high precision measurements. Such data yield poorly defined barrier distributions and can therefore be equally well reproduced with models incorporating very different barrier distributions, as shown by the dashed and solid lines. Thus testing the concept of barrier distributions, which requires the estimation of the second derivative of the experimentally measured quantity $\sigma E$ with respect to $\mathrm{E}$, needs the fusion excitation function to be measured to much higher levels of precision.

This necessity prompted experiments [10] at the Australian National University, where high precision (uncertainty $~ 1 \%$ ) measurements of fusion excitation functions were made. The barrier distribution for the ${ }^{16} \mathrm{O}+{ }^{154} \mathrm{Sm}$ reaction, extracted from these data is shown in Fig. 2(b). The advantages of high precision measurements are clear in defining the experimental distribution at the level where it can now distinguish between the two different calculations. The reactions of ${ }^{16} \mathrm{O}$ with deformed ${ }^{154} \mathrm{Sm}$ and ${ }^{186} \mathrm{~W}$ nuclei were chosen to serve as an experimental test of the barrier distribution concept, since the effects of deformation are well established, and the barrier distributions were expected to be close to the classical ones, which are readily calculable.

The measured [10,11] excitation functions for ${ }^{16} \mathrm{O}+{ }^{154} \mathrm{Sm}$ and ${ }^{186} \mathrm{~W}$ are shown in the upper panels of Fig. 3. The details of the experimental set-up used to obtain these data and the precautions taken to obtain the low experimental uncertainties are described in Ref. [9]. The barrier distributions extracted for these two systems are very different as 
shown in the the lower panels of Fig. 3. The dotted curves are the results of calculations using a single-barrier penetration model. The obvious features are that the experimental cross-sections are underestimated and the experimental distributions are very wide relative to the calculations using a single-barrier. The inclusion of the effects of the static quadrupole deformations of the ${ }^{154} \mathrm{Sm}$ and ${ }^{186} \mathrm{~W}$ nuclei in the calculations (dashed line) dramatically improves the agreement with experiment, producing a wide barrier distribution, arising from the random orientations of the deformed nuclei. In fact comparing the calculated and experimental excitation functions, as done traditionally, one could be led to believe that they agree very well. The barrier distributions however give a different picture; the calculations disagree with the measurements at E $56 \mathrm{MeV}$ and $66 \mathrm{MeV}$ for ${ }^{154} \mathrm{Sm}$ and ${ }^{186} \mathrm{~W}$ respectively and they do so in different ways for the two reactions. This suggests that the data are sensitive to nuclear properties other than the quadrupole deformation.

The ${ }^{154} \mathrm{Sm}$ and ${ }^{186} \mathrm{~W}$ nuclei are known to have significant hexadecapole deformations which are similar in magnitude but differ in sign; the former has a positive $\beta_{4}$ and the latter a negative one. The results of including the effects of hexadecapole deformations in the calculations are shown by the solid lines, which agree remarkably well with the experimental results. It is clear that the differences in the shape of the calculated (and experimental) barrier distributions for the two systems is mainly due to the differences in the sign of the $\beta_{4}$ value. Some minor discrepancies between these calculated and the experimental barrier distributions which are still present, are discussed in Ref. [9].

The results of these measurements exceeded all expectations, showing that fusion excitation functions are not only very sensitive to quadrupole deformations but also to small hexadecapole deformations as well. These measurements also made it possible to apply the proposition of extracting fusion barrier distribution from the experimental data, and showed that for permanently deformed nuclei the function $d^{2}(E \sigma) / d E^{2}$ does indeed return a sensible representation of barriers expected to be encountered during the reaction.

\section{VIBRATIONAL NUCLEI - DOES THE CONCEPT STILL WORK ?}

The barrier distributions extracted for the rotational nuclei provided a good test of this new approach to fusion. Since for these cases the concept of a "distribution" had a classical analogue, it was crucial to test whether this picture would still be applicable in cases where the existence of a distribution of barriers is not classically apparent. Further, the concept of a distribution is strictly valid for cases with zero excitation energy (eigenchannel approximation) and thus it was not clear whether couplings to vibrational states with typical excitation energies of a few $\mathrm{MeV}$ will give rise to the simple barrier distribution as predicted in Ref. [4]. The reactions ${ }^{16,17} \mathrm{O}+{ }^{144} \mathrm{Sm}$ were chosen for this purpose as the coupling scheme is expected to be relatively simple, because the degree of collectivity associated with the semi-magic nucleus ${ }^{144} \mathrm{Sm}$ is relatively small. The use of ${ }^{17} \mathrm{O}$ was to check if the distributions are sensitive to the additional neutron in the projectile.

The measured [12] excitation functions along with the extracted barrier distributions for the ${ }^{16,17} \mathrm{O}+{ }^{144} \mathrm{Sm}$ reactions are shown in the upper and lower panels of Fig. 1 . Calculations assuming no coupling are shown by the dotted lines; clearly they are inconsistent with experiment. The experimental barrier distributions for both reactions are domi- 
nated by a two-peaked structure, with the main strength around $60 \mathrm{MeV}$ and a peak with smaller strength near $65 \mathrm{MeV}$; this picture closely resembles that of Fig. 1(d) , a typical case of coupling to $-\mathrm{Q}$ value channels. Since the two reactions have significantly different $Q$-values for one- and two-particle transfer reactions, and the projectiles have different structure, the consistency of this feature strongly implies that it is associated with inelastic excitation of ${ }^{144} \mathrm{Sm}$. The most important channels associated with excitation of ${ }^{144} \mathrm{Sm}$ are expected to involve those states with the largest $\mathrm{B}(\mathrm{E} \lambda) \uparrow$ values, i.e. the first $2^{+}$and $3^{-}$states. Considering firstly the ${ }^{16} \mathrm{O}$ reaction, including the $2^{+}$and $3^{-}$ states of ${ }^{144} \mathrm{Sm}$ in the calculation gives a good representation [9] of the data, as shown in Fig. 四(a) and (b).

In the reaction with the ${ }^{17} \mathrm{O}$ projectile, the excitation function and the barrier distribution at higher energies are very similar to those of the reaction with ${ }^{16} \mathrm{O}$, but at low energies the cross-sections for the ${ }^{17} \mathrm{O}$ reaction are more than four times higher than those for ${ }^{16} \mathrm{O}$. A calculation including couplings to the $2^{+}$and $3^{-}$states of ${ }^{144} \mathrm{Sm}$, similar to that which describes the ${ }^{16} \mathrm{O}$ reaction very well, shown by the dashed line in Fig. 四(c) and (d), fails to fit the low energy data. It is clear from the barrier distribution that this calculation gives too much strength around the main peak and fails to reproduce the observed low energy tail, indicating a need for additional coupling. An increased crosssection will result from any form of extra coupling, as was shown in Fig. 1, but the type of coupling required is not immediately apparent in the excitation function. However, referring to Fig. 1 (e), a low energy tail in the barrier distribution would imply couplings to a + Q-value channel, which rules out couplings to further inelastic channels, and indicates couplings to transfer channels. The neutron stripping reaction ${ }^{144} \mathrm{Sm}\left({ }^{17} \mathrm{O},{ }^{16} \mathrm{O}\right){ }^{145} \mathrm{Sm}$ has a ground-state $Q$-value of $+2.6 \mathrm{MeV}$ and is the obvious candidate for inclusion in the calculations. The calculations including couplings to this transfer channel give an extremely good representation of the data [9] as shown by the solid line in the right panels of Fig. 14.

The excellent agreement between the theoretical calculations and the experimental results strongly supports the barrier distribution picture, even when the excitation energies are not very close to zero as in the eigenchannel picture, thus indicating that this technique can potentially be used as a tool to give a better understanding of more complex reaction processes. These experiments also unambiguously demonstrate the effects on fusion of couplings to inelastic and transfer channels. Since these experiments, measurements for the reactions ${ }^{32,36} \mathrm{~S}+{ }^{110} \mathrm{Pd}$ and ${ }^{40} \mathrm{Ca}+{ }^{46,48,50} \mathrm{Ti}$ have been studied at other laboratories [13,14] and have nicely demonstrated the effects of couplings to transfer channels on the barrier distributions.

\section{UNRAVELLING COMPLEX PHONON COUPLINGS}

The successful interpretation of the fusion excitation function for the above-mentioned systems and consequently the clear identification of the couplings affecting fusion prompted further experiments to see and/or identify how more exotic forms of couplings affect the

fusion process. A study [15] of the ${ }^{58} \mathrm{Ni}+{ }^{60} \mathrm{Ni}$ reaction, described below, provided a surprising and striking result due to the effects of complex surface vibrations in fusion. Experimental studies [16] of the fusion of two doubly closed shell nuclei, the ${ }^{16} \mathrm{O}+$ ${ }^{208} \mathrm{~Pb}$ system, yielded yet another surprising result which is discussed in Sec. 5.2. 


\subsection{The ${ }^{58} \mathrm{Ni}+{ }^{60} \mathrm{Ni}$ reaction}

This system was studied [15] at the Laboratori Nazionali di Legnaro, Italy, in order to address the question of whether sub-barrier fusion enhancement is mainly due to an elastic $(Q=0)$ two neutron transfer channel, or due to couplings to vibrational states in the two $\mathrm{Ni}$ isotopes. The results have been discussed in detail by $\mathrm{N}$. Rowley in this conference and hence here we quote the main results only. It was found that the barrier distribution had a three-peaked structure, a feature which could not be reproduced by coupling to neutron-pair transfer and/or coupling to simple 1-phonon states in the Ni nuclei. It was shown [15] that couplings not only to the 1-phonon states but more importantly to 2 -phonon states in each nucleus (and their mutual excitations) were necessary to obtain a good representation of the data. This was the first time the effects of such complex couplings were clearly identified as affecting the fusion process.

\subsection{The ${ }^{16} \mathrm{O}+{ }^{208} \mathrm{~Pb}$ reaction}

It might be believed a priori that the results of fusion of two doubly closed shell nuclei should be easily explained. Interestingly, previous [17] experiments and analyses encountered difficulties in explaining the fission fragment anisotropies and the deduced fusion mean square angular momentum. High precision fusion cross-section measurements were made [16] with the aim of understanding some of these features. The measured fusion excitation function and the barrier distribution are presented in Fig. 5. The single-barrier penetration calculations (dashed lines) underpredict the excitation function, and fail to reproduce the wide barrier distribution, clearly indicating the presence of couplings with other channels.

The solid lines in the left panels of Fig. 5 show the results of a realistic coupledchannels calculation [18] which includes couplings to the $2^{+}, 3^{-}$and $5^{-}$vibrational states in ${ }^{208} \mathrm{~Pb}$ (discussions regarding the non-inclusion of transfer channels is detailed in Ref. [19]). The calculations predict a double-peaked structure as opposed to the more complex structure seen in the experimental barrier distribution, and also underpredict the low energy part of the excitation function. It can easily be shown 20 that the doublepeaked structure of the calculated barrier distribution will remain essentially unchanged even when couplings to other 1-phonon states are considered. Neither can the agreement be improved by increasing the coupling strength. It is thus clear that couplings from the elastic channel to single-phonon states in ${ }^{208} \mathrm{~Pb}$ are not sufficient to explain the data.

Using an eigenchannel approximation it has been shown [20] that the introduction of couplings to 2-phonon states results in three barriers, with the two lower barriers being close to each other and the third barrier lying at higher energy. In the present case, the experimental barrier distribution would seem to indicate this scenario. The existence of 2 -phonon octupole excitations in ${ }^{208} \mathrm{~Pb}$ was recently shown 21] experimentally. Results of the coupled-channels calculations, including the $2^{+}, 3^{-}, 3^{-} \otimes 3^{-}$and $5^{-}$vibrational states in ${ }^{208} \mathrm{~Pb}$, and all the resulting cross-coupling terms e.g., $2^{+} \otimes 3^{-}$etc., are shown by the solid lines in the right panels of Fig. 5. It is clear that the agreement with the experimental excitation function and the shape of the barrier distribution is dramatically improved [19] with the inclusion of couplings to 2-phonon states.

These experimental results thus support the recent spectroscopic measurements indi-

cating the presence of a double-phonon state in ${ }^{208} \mathrm{~Pb}$. It is interesting that the dynamics 
of the fusion process even for reactions between two closed shell nuclei, which might be thought to be simple, particularly at low energies, is affected by complex surface vibrations.

\section{ROLE OF CLOSED SHELL NUCLEI}

As seen in the previous sections, a successful description of the fusion cross-sections for reactions involving ${ }^{16} \mathrm{O}$ did not require couplings to states in ${ }^{16} \mathrm{O}$. This is particularly curious as the octupole vibrational state has a large strength and indeed previous work 22,23] on the ${ }^{16} \mathrm{O}+{ }^{154} \mathrm{Sm},{ }^{A} \mathrm{Ge}$ reactions included couplings to this state. In marked contrast, no specific features appear in the measured barrier distribution for the ${ }^{16} \mathrm{O}+{ }^{144} \mathrm{Sm}$ reaction which can be associated with the excitation of ${ }^{16} \mathrm{O}$; rather it was seen [9] that a good theoretical representation of the experimental fusion barrier distribution is destroyed when the projectile excitation is included. Recent measurements of ${ }^{40} \mathrm{Ca}+{ }^{194} \mathrm{Pt},{ }^{192} \mathrm{Os}$ [24] at the University of Washington, Seattle, show a characteristic structure with a high energy peak arising due to coupling to the octupole state of ${ }^{40} \mathrm{Ca}$, as indicated by the vertical arrows in Fig. 6.

Both of these conclusions are based on the comparison of the experimental data with the results of simplified coupled-channels calculations [25,26], where the linear coupling approximation is used to describe the vibrational excitation of the projectile. The inadequacy of the linear coupling approximation has been pointed out by several authors [18,27, and it is likely that this approximation does not give the true fusion barrier distribution for couplings to the $3^{-}$state in ${ }^{16} \mathrm{O}$, which has a large E3 strength. The results of realistic coupled-channels calculation [18] under the linear coupling approximation, for the ${ }^{16} \mathrm{O}+{ }^{144} \mathrm{Sm}$ reaction with and without couplings to the $3^{-}$state in ${ }^{16} \mathrm{O}$ are shown in the left panels of Fig. 7. It is seen that couplings to the $3^{-}$state in ${ }^{16} \mathrm{O}$ gives essentially a single-peaked barrier distribution and destroys the good agreement with the data obtained by ignoring it, as also observed in Ref. [9].

Performing similar realistic calculations, where however the couplings to the octupole vibrations of both ${ }^{16} \mathrm{O}$ and ${ }^{144} \mathrm{Sm}$ are treated to all orders, gives the result [28] shown in the right hand panels of Fig. 7. It is remarkable that these calculations re-establish the double-peaked structure seen in the experimental data, which was missing in the linear coupling calculations. Indeed, the barrier distribution obtained by including the coupling to the octupole vibration of ${ }^{16} \mathrm{O}$ to all orders, looks very similar to that obtained by totally ignoring it, apart from a shift in energy. A shift of $2 \mathrm{MeV}$ (dashed line) of the former is required for the two calculated distributions to coincide. This shift is consistent with the general conclusion that the main effect of the coupling to an inelastic channel whose excitation energy is larger than the curvature of the bare fusion barrier, i.e. an adiabatic coupling, is to introduce a static potential shift [29]. Thus the shape of the barrier distribution does not change unless the coupling is very strong and the coupling form factor itself has a strong radial dependence. The effects of these excitations can then be included in the 'bare' potential in the coupled-channels calculations. Thus for ${ }^{16} \mathrm{O}+{ }^{144} \mathrm{Sm}$, where potential parameters for the calculations are obtained from a fit to the high energy data, the effects of the octupole vibration of ${ }^{16} \mathrm{O}$ are already included. The explicit inclusion of the coupling to octupole vibration then leads to double counting which 
manifests itself by introducing an additional shift in the barrier (or barrier distribution) as observed.

\section{APPLICATIONS}

The improved understanding of the fusion process as a result of the new measurements can lend itself to applications in other fields, e.g. population of high spin states for nuclear spectroscopy studies and formation of super-heavy elements. These aspects were discussed in the previous talk by N. Rowley and here we will limit ourselves to a discussion of the anomalous fission fragment anisotropies, which were explained in terms of our knowledge of the fusion barrier distributions.

\subsection{Nuclear orientation effects in fission}

The angular distribution for the fission process is characterised by the anisotropy, defined as the ratio of yield at $180^{\circ}$ to that at $90^{\circ}$ with respect to the beam axis $\mathrm{W}\left(180^{\circ}\right) / \mathrm{W}\left(90^{\circ}\right)$. The anisotropies generally decrease with decreasing bombarding energies. However, it was observed [17,30 that for reactions with actinide targets at nearbarrier energies, the anisotropies were not only much larger than the predictions of the transition state model (TSM) [31], but increased as the beam energy dropped through the fusion barrier region. This observation agrees with the recently measured [32] anisotropies for the ${ }^{16} \mathrm{O}+{ }^{238} \mathrm{U}$ shown in the lower panel of Fig. 8. The expected energy dependence of the anisotropy based on the TSM calculation is indicated by the dot-dashed line. No reasonable variation of parameters can reproduce the data. It is clear that the observed anisotropies cannot be described by this model.

There exists a striking correlation between anisotropy and the barrier distribution; the low barriers are correlated with the higher anisotropies and the transition from higher to lower anisotropies occur at an energy close to the main peak. This observation prompted [32] a simple geometrical model to be proposed which is able to explain the data in a simple and elegant way. Considering the collision classically, the higher fusion barriers correspond to contact of the projectile with the flattened side of the prolate target, resulting initially in a compact dinuclear system. Conversely, the lower barriers correspond to contact with the tip, giving an elongated dinuclear system. Intuitively, it seems reasonable that the former configuration would be more likely to result in fusionfission, and the latter in quasi-fission (fission-like fragments from a system which never formed a compact compound nucleus inside the unconditional fission barrier).

It was assumed that collisions with the tips of the target nuclei result in quasi-fission only. The assumed energy dependence of the anisotropies for quasi-fission is shown by the dotted line in Fig 8. The anisotropy for fusion-fission is taken from the transition state model, and is shown by the dot-dashed line in the figure. The average anisotropy, shown by the solid line in the figure, was determined by taking the anisotropy assumed for quasifission, and the fusion-fission anisotropies calculated with the transition state model, and weighting each of them appropriately with a geometrical factor [33]. The calculations show that as we go from lower to higher energies there is a gradual transition from anisotropies due to quasi-fission to those due to a mixture of quasi-fission and fusion-fission. The calculations follow the trends of the data very well, resolving the observed anomaly and supporting the hypothesis that collisions with the tips of the deformed target nuclei result 
in quasi-fission, whilst collisions with the sides result in fusion, followed almost always by fission. This mechanism may have significance in explaining the failure of reactions with actinide targets to give larger cross-sections for the formation of very heavy nuclei than cold fission reactions with targets near $\mathrm{Pb}$.

\section{Summary}

High precision measurements of fusion excitation functions have challenged the view that fusion cross-sections are smooth functions of energy which can be reproduced by any model which include some couplings. These measurements, which enable the extraction of a representation of the barriers encountered during the fusion process, provide an identification not only of the main couplings, but also the way in which they affect fusion. With this improved understanding of the fusion process we now may predict the dominant couplings likely to be present in a given reaction, benefiting fields which use fusion as a tool. The study of fusion reactions close to the barrier investigates features of couplingassisted tunnelling, a phenomenon appearing in a diverse range of physical systems. It would be interesting to see whether one can exploit the ability in nuclear physics to vary the coupling strength and learn more about such problems.

\section{REFERENCES}

1. S.G. Steadman and M.J. Rhoades-Brown, Ann. Rev. Nucl. Part. Sci. 36 (1986) 649 and references therein.

2. M. Beckerman, Rep. Prog. Phys. 51 (1988) 1047 and references therein.

3. A.B. Balantekin, S.E. Koonin, and J.W. Negele, Phys. Rev. C28 (1983) 1565.

4. C.H. Dasso, S. Landowne and A. Winther, Nucl. Phys. A 405 (1982) 381; 407 (1983) 221.

5. C.Y. Wong, Phys. Rev. Lett. 31 (1973) 766.

6. L.C. Vaz and J.M. Alexander, Phys. Rev. C 10 (1974) 464.

7. R.G. Stokstad, Y. Eisen, S. Kaplanis, D. Pelte, U. Smilansky, and I. Tserruya, Phys. Rev. Lett. 41 (1978) 465; Phys. Rev. C 21 (1980) 2427.

8. N. Rowley, G.R. Satchler, and P.H. Stelson, Phys. Lett. B 254 (1991) 25.

9. J.R. Leigh, M. Dasgupta, D.J. Hinde, J.C. Mein, C.R. Morton, R.C. Lemmon, J.P. Lestone, J.O. Newton, H. Timmers, J.X. Wei, and N. Rowley, Phys. Rev. C 52 (1995) 3151.

10. J.X. Wei, J.R. Leigh, D.J. Hinde, J.O. Newton, R.C. Lemmon, S. Elfström, J.X. Chen, and N. Rowley, Phys. Rev. Lett. 67 (1991) 3368.

11. R.C. Lemmon, J.R. Leigh, J.X. Wei, C.R. Morton, D.J. Hinde, J.O. Newton, J.C. Mein, and M. Dasgupta, Phys. Lett. B 316 (1993) 32.

12. C.R. Morton, M. Dasgupta, D.J. Hinde, J.R. Leigh, R.C. Lemmon, J.P. Lestone, J.C. Mein, J.O. Newton, H. Timmers, N. Rowley, and A.T. Kruppa, Phys. Rev. Lett. 72 (1994) 4074.

13. A.M. Stefanini, D. Ackermann, L. Corradi, J.H. He, G. Montagnoli, S. Beghini, F. Scarlassara, and G.F. Segato, Phys. Rev. C 52 (1995) R1727.

14. R. Vandenbosch, A.A. Sonzogni, and J.D. Bierman, to appear in J. Phys. G (1997).

15. A.M. Stefanini, D. Ackermann, L. Corradi, D.R. Napoli, C. Petrache, P. Spolaore, P. 
Bednarczyk, H.Q. Zhang, S. Beghini, G. Montagnoli, L. Mueller, F. Scarlassara, G.F. Segato, F. Soramel, and N. Rowley, Phys. Rev. Lett. 74 (1995) 864.

16. C.R. Morton, D.J. Hinde, J.R. Leigh, J.P. Lestone, M. Dasgupta, J.C. Mein, J.O. Newton, and H. Timmers, Phys. Rev. C 52 (1995) 243.

17. T. Murakami, C.-C. Sahm, R. Vandenbosch, D.D. Leach, A. Ray, and M.J. Murphy, Phys. Rev. C 34 (1986) 1353.

18. K. Hagino, N. Takigawa, M. Dasgupta, D.J. Hinde, J.R. Leigh, Phys. Rev. C 55 (1997) 276.

19. M. Dasgupta, K. Hagino, C.R. Morton, D.J. Hinde, J.R. Leigh, N. Takigawa, H. Timmers, and J.O. Newton, to appear in J. Phys. G (1997).

20. N. Rowley and M. Dasgupta, Proc. Int. Conf. on Heavy-Ion Reactions with Radioactive Beams (RIKEN) (Singapore: World Scientific Press) p 232, 1993.

21. Minfang Yeh, P.E. Garrett, C.A. McGrath, S.W. Yates, and T. Belgya, Phys. Rev. Lett. 76 (1996) 1208.

22. P.R.S. Gomes, I.C. Charret, R. Wanis, G.M. Sigaud, V.R. Vanin, R. Liguori Neto, D. Abriola, O.A. Capurro, D.E. DiGregorio, M. di Tada, G. Duchene, M. Elgue, A. Etchegoyen, J.O. Fernández Niello, A.M.J. Ferrero, S. Gil, A.O. Macchiavelli, A.J. Pacheco, and J.E. Testoni, Phys. Rev. C49 (1994) 245.

23. E.F. Aguilera, J.J. Kolata, and R.J. Tighe, Phys. Rev. C 52 (1995) 3103.

24. J.D. Bierman, P. Chang, J.F. Liang, M.P. Kelly, A. A. Sonzogni, and R. Vandenbosch, Phys. Rev. Lett. 76 (1996) 1587.

25. C.H. Dasso and S. Landowne, Comp. Phys. Commun. 46 (1987) 187; J.O. Fernández Niello, C.H. Dasso, and S. Landowne, ibid. 54 (1989) 409.

26. M. Dasgupta, A. Navin, Y.K. Agarwal, C.V.K. Baba, H.C. Jain, M.L. Jhingan, and A. Roy, Nucl. Phys. A 539 (1992) 351.

27. H. Esbensen and S. Landowne, Phys. Rev. C 35 (1987) 2090.

28. K. Hagino, N. Takigawa, M. Dasgupta, D.J. Hinde, J.R. Leigh, to appear in Phys. Rev. Lett. (1997).

29. N. Takigawa, K. Hagino, M. Abe and A.B. Balantekin, Phys. Rev. C 49 (1994) 2630.

30. H. Zhang, Z. Liu, J. Xu, X. Qian, Y. Qiao, C. Lin, and K. Xu, Phys. Rev. C 49 (1994) 926, and references therein.

31. R. Vandenbosch and J.R. Huizenga, Nuclear Fission, (Academic Press NY) 1973.

32. D.J. Hinde, M. Dasgupta, J.R. Leigh, J.P. Lestone, J.C. Mein, C.R. Morton, J.O. Newton, and H. Timmers, Phys. Rev. Lett. 74 (1995) 1295.

33. D.J. Hinde, M. Dasgupta, J.R. Leigh, J.C. Mein, C.R. Morton, J.O. Newton, and H. Timmers, Phys. Rev. C 53, (1996) 1290. 
Figure 1. Calculated excitation functions and barrier distributions for a single-barrier (dashed lines) compared with three coupling schemes involving coupling to a negative $Q$ value channel (a) and (d), a positive $Q$-value channel (b) and (e), and coupling associated with a nucleus with a permanent quadrupole deformation (c) and (f). The cross-sections, plotted against the ratio of the energy to the average barrier $B_{0}$, are essentially identical at low and high energies. The type of coupling is more easily seen in the lower part of the figure than in the excitation functions themselves. (Adapted from Ref. 9).

Figure 2. Precise excitation functions are necessary to obtain meaningful barrier distributions. Two barrier distribution are shown, extracted from data with uncertainties of (a) $10 \%$ and (b) $1 \%$. The data in panel (a) is unable to distinguish between the two calculations shown by the dashed and solid lines. 
Figure 3. Comparison of the measured fusion excitation functions and the extracted barrier distributions for the indicated reactions. The dotted curves are the result of singlebarrier penetration calculations. The dashed lines show the calculations with the inclusion of the quadrupole deformations of ${ }^{154} \mathrm{Sm}$ and ${ }^{186} \mathrm{~W}$; the solid lines include hexadecapole deformations. The differences in the shape of the barrier distribution is essentially due to the different sign of the hexadecapole deformations for the two nuclei. (From Ref. 9).

Figure 4. Comparison of the measured excitation functions (a and c) and extracted barrier distributions ( $b$ and $\mathrm{d}$ ) for ${ }^{16} \mathrm{O}+{ }^{144} \mathrm{Sm}$ and for ${ }^{17} \mathrm{O}+{ }^{144} \mathrm{Sm}$. Couplings to the $2^{+}$ and $3^{-}$states of ${ }^{144} \mathrm{Sm}$ is adequate to describe the ${ }^{16} \mathrm{O}$ data as shown by the solid lines in the left panels. These couplings are however not sufficient for the ${ }^{17} \mathrm{O}$ data (dashed lines in the right panels), which requires additional coupling to a neutron transfer channel with positive $Q$-value (solid lines).

Figure 5. Comparison of the experimental data with calculations including couplings to one-phonon states (left panels) and up to two-phonon states in ${ }^{208} \mathrm{~Pb}$ (right panels).

Figure 6. Experimental fusion barrier distributions for the ${ }^{40} \mathrm{Ca}+{ }^{192} \mathrm{Os},{ }^{194} \mathrm{Pt}$ reactions; the arrow indicates the feature arising as a result of coupling to the $3^{-}$state in ${ }^{40} \mathrm{Ca}$. Data are from Ref. 24.

Figure 7. Fusion excitation function and the barrier distribution for the ${ }^{16} \mathrm{O}+{ }^{144} \mathrm{Sm}$ system compared with realistic coupled-channels calculations with the linear coupling approximation (left panels) and all order coupling (right panels). The solid lines are the results when ${ }^{16} \mathrm{O}$ is treated as inert. The dashed lines are the results when the coupling to the octupole vibration of ${ }^{16} \mathrm{O}$ is also taken into account; the long-dashed line in the right panel is the same calculation as the dashed line, with but with the average barrier increased by $2 \mathrm{MeV}$. Effects of the octupole vibration of ${ }^{144} \mathrm{Sm}$ are taken into account in all the calculations. (Adapted from Ref. 28).

Figure 8. Panel (a) shows the experimental fusion barrier distribution for the ${ }^{16} \mathrm{O}$ $+{ }^{238} \mathrm{U}$ reaction. The effects of static deformation of ${ }^{238} \mathrm{U}$ have been included in the calculations shown by the solid line. The fission anisotropies are shown in panel (b). The expected anisotropy based on the statistical model calculation is indicated by the dot-dashed line, whilst the assumed quasi-fission anisotropy is given by the dotted line. The model described in the text gives the solid curve. 